\title{
Accuracy of Determining Stress Intensity Factors in Some Numerical Programs
}

\author{
M. Vorel, E. Leidich
}

At present, there are many programs for numerical analysis of cracks, in particular for determining stress intensity factors. Analyses of a single-edge cracked beam and flat plate with a semielliptical surface crack are presented in this study to examine the accuracy and applicability of the Franc2d and Franc3d programs. Further numerical computations of the MARC program and analytical solutions of stress intensity factors were included to compare the results with each other. For this purpose MARC was equipped with special user procedures. The influence of mesh fineness on the results was also investigated in all programs. The distributions of the stress intensity factors show good agreement in quality. The maximum deviations from the analytical solutions are $9.7 \%$. With greater numbers of elements programs Franc2d and Franc3d showed some instability, which currently reduces the usefulness and reliability of these promising tools for engineering applications.

Keywords: fracture mechanics, stress intensity factors, numerical programs.

\section{Introduction}

Stress intensity factors can be determined by experimental, numerical or analytical methods. However, with complicated component and crack geometry or under complex loading only numerical procedures are applicable. Many programs have been designed recently to deal with fracture phenomena (e. g., Franc2d, Franc/FAM, Franc3d and $A F G R O W$ ), which, in spite of great programming efforts, still show some deficiencies in range of functionality, operation comfort and reliability. Little evidence has yet been provided about the accuracy and suitability of such programs for solving engineering problems [1, 2]. Present applications cover illustrative examples and simple problems $[3,4,5]$.

Proven multi-purpose numerical programs such as $M A R C$ and $A B A Q U S$ usually possess no routines to find out stress intensity factors. To take advantage of the programs' extensive functions and high reliability a number of user subroutines for solving stress intensity factors need to be programmed.

As regards the accuracy of stress intensity factors, only single programs have been compared with analytical solutions so far, and no comparison of the programs with each other has been presented. The objective of this study was therefore to confront the results of Franc $2 d$, Franc $3 d$ and $M A R C$ with each other and for a better view also to verify their deviations from the analytical solutions. The analyses were conducted on simple models while also observing the influence of mesh fineness, usability of available solvers and the overall performance of the programs.

\section{Programs and models}

The tested programs were Franc2d Version 2.7 [6], Franc3d [7] Version 1.15, and MARC Version 2000 [8]. The first two come from Cornell University, New York, and as freeware they can be freely distributed. Franc2d (two-dimensional FRActure ANalysis Code) is based on the finite element method and enables analyses of two-dimensional problems with arbitrary component and crack geometries. Several methods are implemented for calculating stress intensity factors, from which the J-Integral Method [9] was chosen for the purposes of this study. Franc3d uses the boundary element method and was designed for solving three-dimensional fracture problems. Also here, arbitrary component and crack geometries can be analysed. Stress intensity factors are determined by the Displacement Correlation Method [10]. Both Franc2d and Franc3d possess further important functions for modeling various fracture phenomena, such as fatigue crack growth. Currently, a new version of Franc $3 d$ is being developed, which is based on the finite element method and offers a greater functional range. The finite element system $M A R C$ is suitable for analyses of general problems of engineering mechanics. To determine stress intensity factors the Displacement Correlation Method with a linear extrapolation from two nodes at each crack face $[11,12]$ has been implemented in the user procedures.

As analytical solutions are known for only certain probe types, a single-edge cracked beam subjected to three-point bending and a flat plate with a semielliptical surface crack (Fig. 1) were chosen for the tests in this study. The analytical solution for the single-edge cracked beam is given by the equation

$K_{I}=\frac{3 F s}{2 B W^{2}} \sqrt{a} \frac{1.99-\frac{a}{W}\left(1-\frac{a}{W}\right)\left[2.15-3.93 \frac{a}{W}+2.7\left(\frac{a}{W}\right)^{2}\right]}{\left(1+2 \frac{a}{W}\right)\left(1-\frac{a}{W}\right)^{\frac{3}{2}}}(1)$

with the conditions

$$
\frac{s}{W}=4, \quad \text { and } \quad 0 \leq \frac{a}{W} \leq 0.6 .
$$

The distribution of stress intensity factors along the crack front in a flat plate is expressed by the relation

$$
K_{I}=\frac{1}{\boldsymbol{\Phi}} \sigma_{n} \sqrt{\pi c} Y\left(\psi, \frac{c}{a}, \frac{c}{W}, \frac{a}{b}\right)
$$

with the conditions

$$
0<\frac{c}{a} \leq 0.5, \quad 0 \leq \frac{c}{W}<1.0 \quad \text { and } \quad 0 \leq \psi \leq \pi,
$$

where $\Phi$ is a complete elliptic integral of the second kind and $\mathrm{Y}$ is a geometric function. However, the function $Y$ is not based on theoretical examinations but on experimental studies [13]. 


\section{(a)}

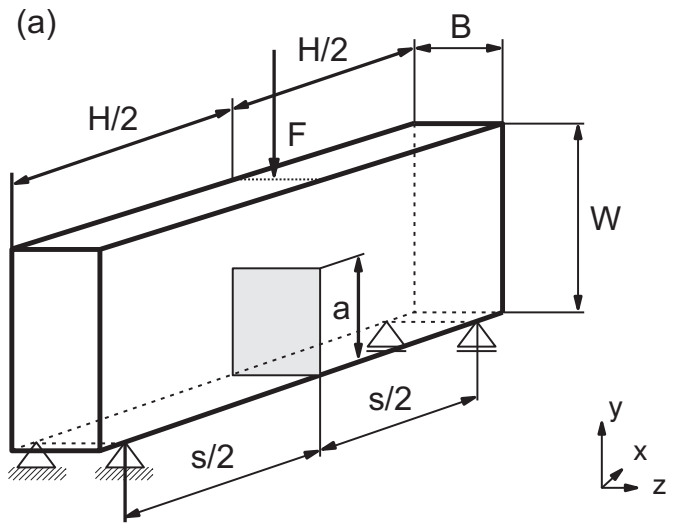

$$
\begin{array}{lll}
W=8 & a=4 & E=210000 \mathrm{MPa} \\
\mathrm{B}=4 & \mathrm{~s} / 2=16 & \mathrm{n}=0.3
\end{array}
$$$$
\mathrm{H} / 2=18
$$

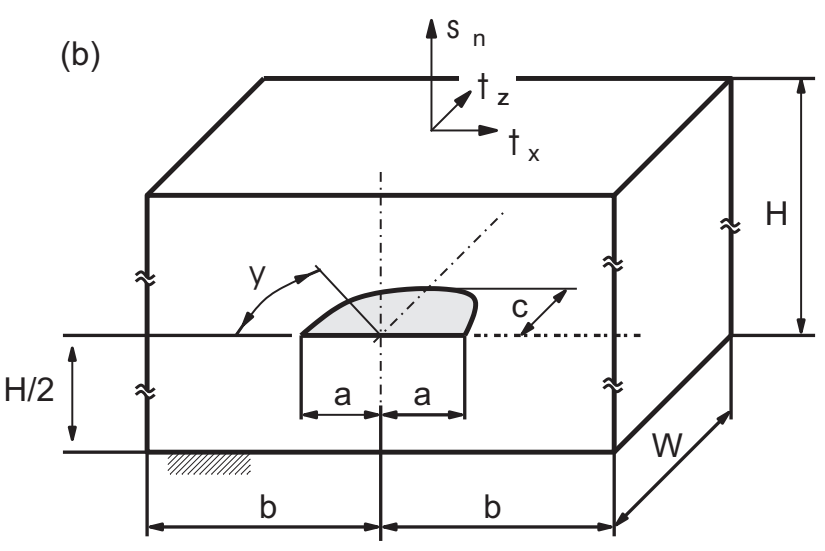

$$
\begin{array}{lll}
H=40 & a=4 & E=210000 \mathrm{MPa} \\
H / 2=20 & c=2 & \mathrm{n}=0.3 \\
\mathrm{~b}=12.566 & \mathrm{~W}=20 &
\end{array}
$$

Fig. 1: A single-edge cracked beam (a) and a flat plate with a semielliptical surface crack (b). Length measures in mm.

The meshes of the analysed models were generated with the relevant preprocessors (Casca, OSM, Mentat) in three mesh densities at a time, in order to observe the influence of mesh fineness on stress intensity factors. Thus, from each examined probe type there were models with a coarse, medium fine and fine mesh in each program (Table 1). Although in general it is appropriate to take advantage of a model symmetry, here, with respect to future studies, complete models were created (Fig. 2). The basic elements were taken linear in Franc3d, and quadratic in Franc2d and MARC. The crack front (or crack tip in two-dimensional cases) formed in all programs collapsed quarter-point quadratic elements [14], the number of which

\begin{tabular}{|c|c|c|c|c|c|c|}
\hline & \multicolumn{3}{|c|}{ Elements } & \multicolumn{3}{|c|}{ Nodes } \\
\hline & coarse & medium fine & fine & coarse & medium fine & fine \\
\hline \multicolumn{7}{|c|}{ Single-Edge Cracked Beam } \\
\hline Franc2d/Casca & 556 & 1636 & 2168 & 1645 & 4525 & 6395 \\
\hline Franc3d/OSM & 936 & 2252 & 4020 & 902 & 2148 & 3876 \\
\hline MARC/Mentat & 2760 & 8400 & 14080 & 13233 & 37939 & 62115 \\
\hline \multicolumn{7}{|c|}{ Centre Cracked Plate } \\
\hline Franc3d/OSM & 1206 & 2014 & 3084 & 1001 & 1918 & 2780 \\
\hline MARC/Mentat & 7396 & 12880 & 17608 & 33143 & 55979 & 76341 \\
\hline
\end{tabular}
varied in three-dimensional cases from 16 to 48 along the crack front. The rosette consisted of 6 to 8 collapsed elements. The two dimensional models were considered as plain strain problems. The meshes of Mentat were generated by a newly introduced parametric modeling function. The load of the single-edge cracked beam models consisted of a sin-

Table 1: Numbers of elements and nodes of the analysed models
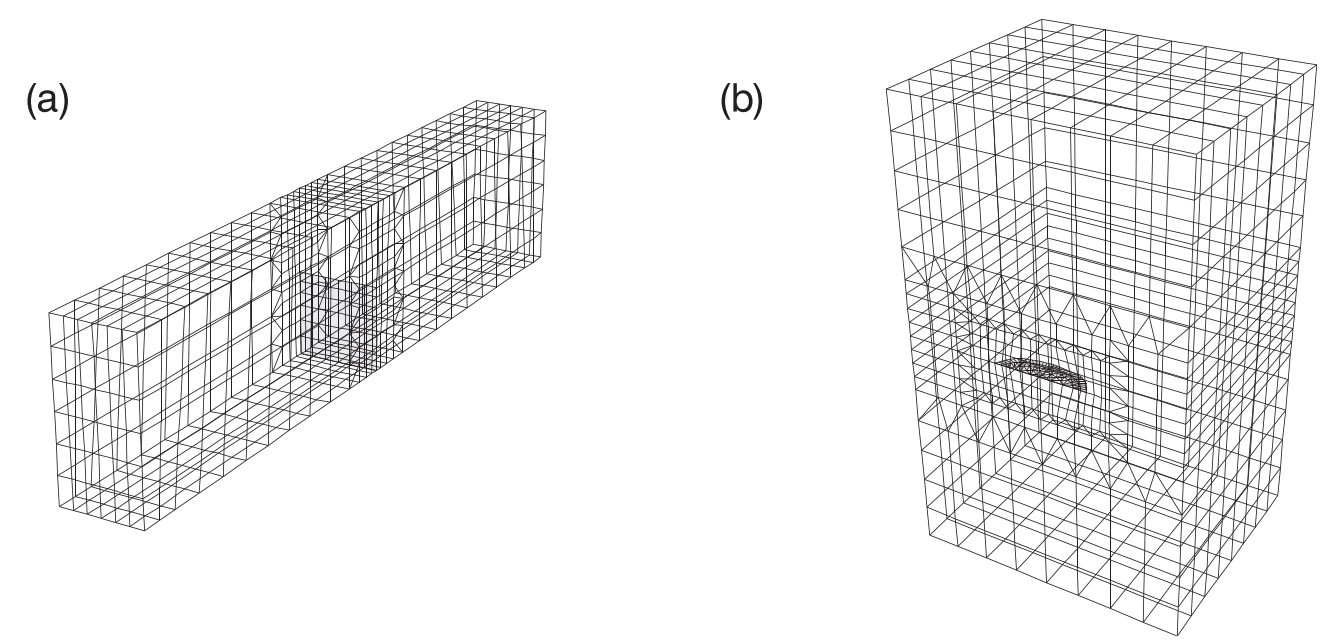

Fig. 2: An example of the used Franc $3 d$ meshes; (a) single-edge cracked beam, (b) flat plate 
gle force $(F=500 \mathrm{~N})$ with two-dimensional models, or of a uniformly distributed force $\left(f=125 \mathrm{Nmm}^{-1}\right)$ in three-dimensional models. The flat plate was loaded with normal stress $\left(\sigma_{n}=200 \mathrm{MPa}\right)$ on the upper surface of the model.

\section{Strategy and results}

A single-edge cracked beam was analysed in Franc $2 d$, Franc3d and MARC. The flat plate was analysed as a sheer three-dimensional problem only in Franc $3 d$ and MARC. The analytical solutions were carried out for both probe types. After analysis there were three results (coarse, medium fine
The dependences of the stress intensity factors on the mesh fineness are displayed in Figs. 4 and 5. The optimum solutions of stress intensity factors are compared in the following diagrams, Fig. 6:

- stress intensity factors in a single-edge cracked beam under single force or distributed force loading; three numerical solutions and one analytical solution; $F=500 \mathrm{~N}$ or $f=125 \mathrm{Nmm}^{-1}$ respectively;

- stress intensity factors in a flat plate under normal stress loading; two numerical solutions and one analytical solution; $\sigma_{\mathrm{n}}=200 \mathrm{MPa}$.

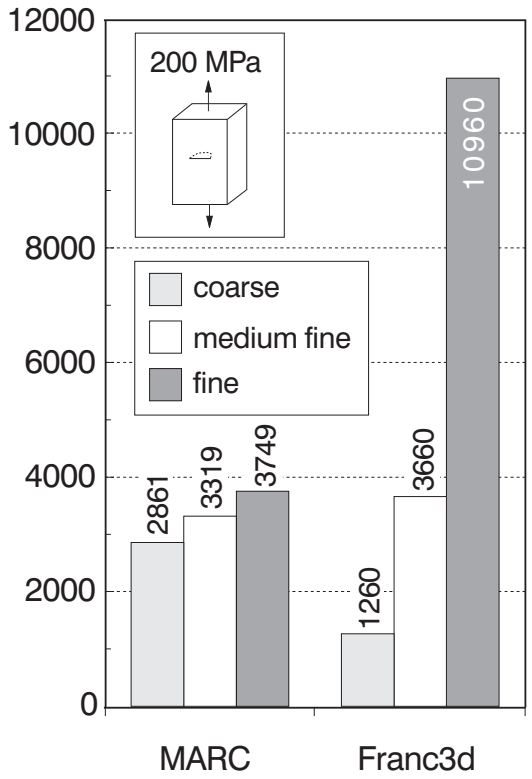

Fig. 3: Computational time of the single-edge cracked beam (left) and flat plate (right)

and fine mesh) for one probe type from each used program. In particular, these were three mesh qualities $\times$ three programs from the single-edge cracked beam, and three mesh qualities $\times$ two programs from the flat plate. From each three results the optimum solutions were then chosen. The criteria for this were minimum deviation from the analytical solution on the one hand, and the lowest possible computational time on the other. In the end the programs' optimum solutions were compared together with the analytical solutions in one diagram for each probe. The stress intensity factors were evaluated as a single value at a crack tip or as a course of values at a crack front.

The Franc2d models were solved with an implicit direct solver, which required very low computational times (Fig. 3). There was no point in using more sophisticated solvers, as the problem was entirely linear. The Franc $3 d$ jobs were processed by the boundary element solver $B E S$, which includes four different schemes. In this study the iterative scheme with out-of-core element storage proved best: the direct scheme could not be applied to larger models, as the programs crashed after the stiffness matrix assembly, and the other schemes turned out to run slightly more slowly. The MARC analyses were carried out with the iterative solver (in-core element storage, incomplete Choleski preconditioner). All computations were performed on an SGI Origin 2000 computer.

\section{Discussion}

With simple crack configurations, such as that of the single-edge cracked beam, mesh quality seems to have only a little influence on the values of the stress intensity factors (Fig. 4). The smallest differences can be observed for Franc2d. However, mesh quality appears to be significant in the case of more complicated crack forms, such as that of a semielliptical crack (Fig. 5). The stress intensity factors differ with MARC especially at the crack edges; Franc $3 d$ shows consistent values from a certain mesh fineness.

The overall comparison of stress intensity factors for a single-edge cracked beam was performed in the middle of the crack front, as these show more important evidence than the values at the crack ends. However, this was not the case for the semielliptical crack in the flat plate, where the stress intensity factors vary considerably along the whole crack front. The values of Franc $3 d$ are always somewhat higher than the analytical solution (deviation up to $9.7 \%$, Fig. 6), whereas $M A R C$ delivers somewhat lower values (deviation up to $8.4 \%$ ). On the one hand, the deviations can be traced to the methods used (boundary element vs. finite element method) and elements (linear vs. quadratic), but on the other hand, they may also result from the different methods of calculating the stress intensity factors. The deviations of the Franc $2 d$ values from the analytical solution are the lowest, which agrees with the 

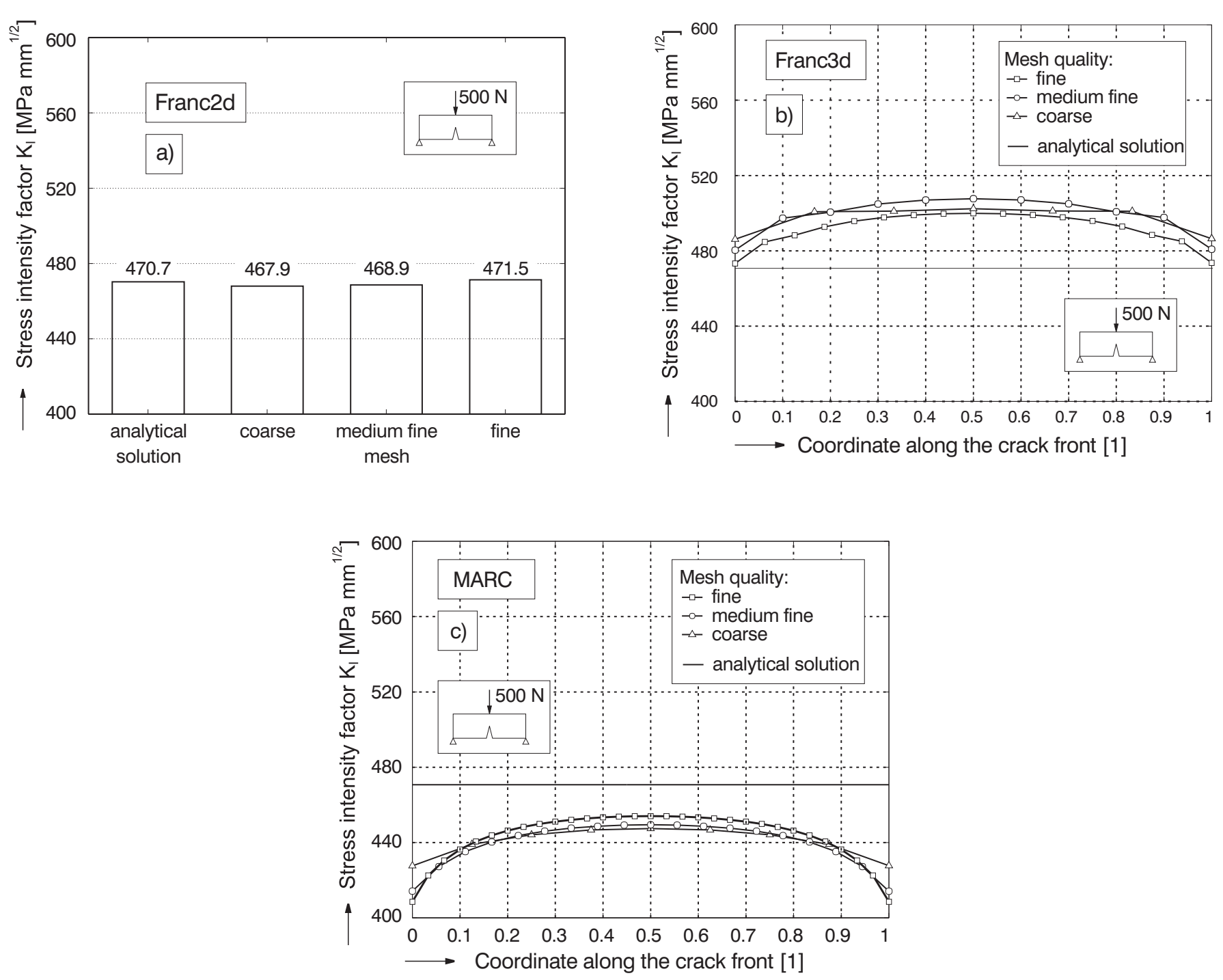

Fig. 4: Influence of mesh fineness on stress intensity factors $\mathrm{K}_{\mathrm{I}}$ for a single-edge cracked beam using Franc2d (a), Franc3d (b) and MARC (c)
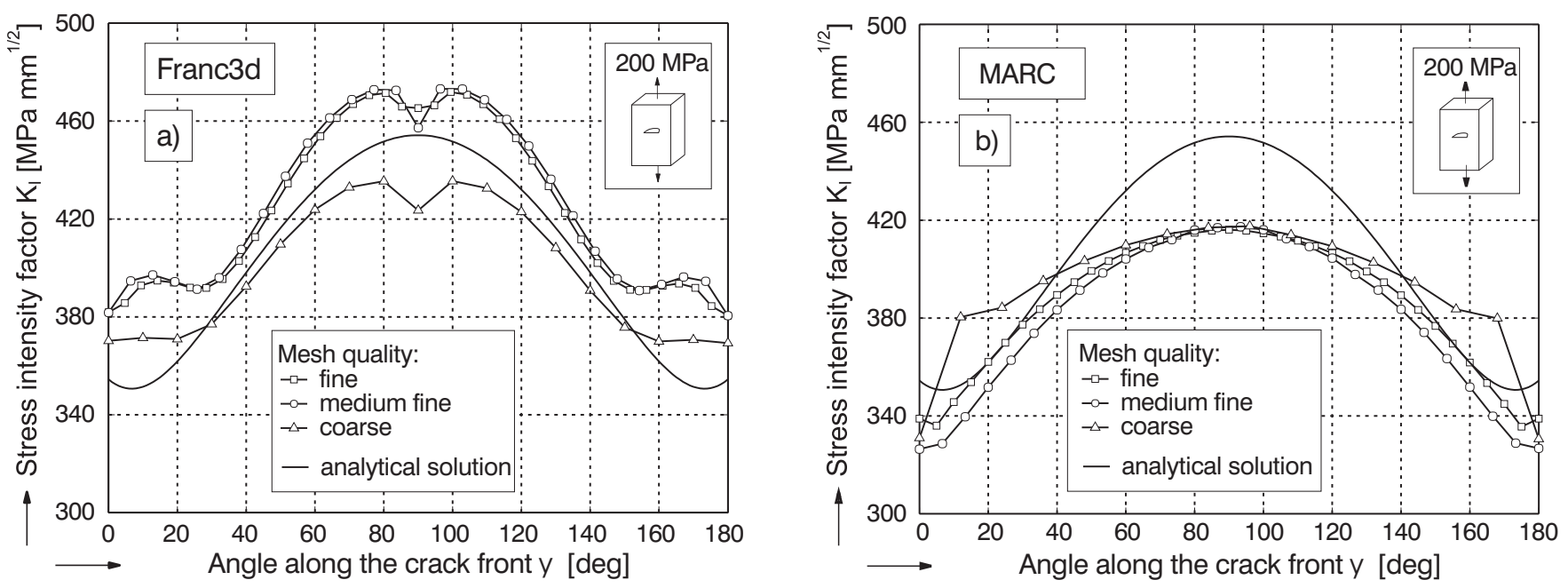

Fig. 5: Influence of mesh fineness on stress intensity factors $K_{\mathrm{I}}$ at the flat plate using Franc3d (a) and $M A R C$ (b)

(c) Czech Technical University Publishing House http://ctn.cvut.cz/ap/ 

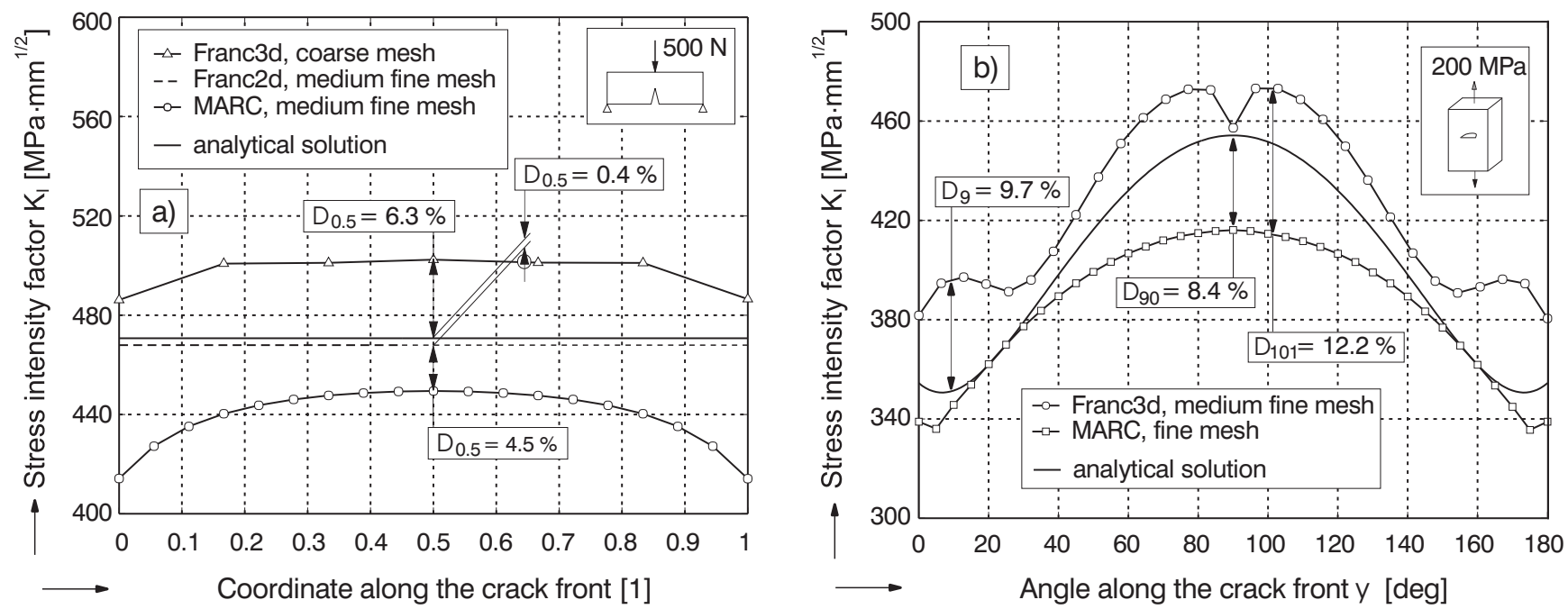

Fig. 6: Comparison of stress intensity factors $\mathrm{K}_{\mathrm{I}}$ for a single-edge cracked beam (a) and flat plate (b)

supposition that domain integral methods (such as the J-Integral Method) are more accurate than local methods (such as the Displacement Correlation Method) [15, 16].

The stress intensity factors determined by Franc $3 d$ at the crack edges in the flat plate do not correspond well to the other displayed solutions (Fig. 6). Stress intensity factors at the crack edges are in general dependent especially on the geometric configuration [13], but they should not acquire such a falling form. False values at crack edges are generally caused by some unsuitable treatment of the singularities which always exist at the ends of a crack front [17]. Here, this phenomenon as well as the sudden decline of the stress intensity factors at $\psi=90^{\circ}$ may also be connected to the boundary element method that is used.

Franc $2 d$ and Franc $3 d$ show some errors during both manual operation and computational processing. These emerge mostly with larger models (from about 4000 elements) and result in disfunction of some commands (e. g., manual redefining of elements). This is probably caused by deficient memory management: during computational processing too high memory demand and falsely defined elements can lead to a program crash.

\section{Conclusion}

In this study some analyses with Franc2d, Franc3d and $M A R C$ were conducted to compare the accuracy of determining stress intensity factors and to examine the behavior of the programs. A single-edge cracked beam and a flat plate with a semielliptical surface crack were used as test models. Franc $2 d$ shows good accuracy, but it is applicable only to two-dimensional problems. Franc3d delivers acceptable values and appears to be a promising tool for engineering applications. To this end, reliability and function range should be improved. MARC with special user procedures shows lower but certainly usable values. Although this multi-purpose program shows high reliability, the programming effort to adapt it for solving fracture problems remains high.

\section{References}

[1] May, B.: Ein Beitrag zur praxisnahen Simulation der Ausbreitung von Ermüdungsrissen bei komplexer Beanspruchung. Düsseldorf, VDI Verlag, 1998

[2] FRANC3D. Documentation: Volume V, Validation/ Verification, New York, Cornell University, 1998

[3] Schöllmann, M., Richard, H. A.: Franc/FAM - A software system for the prediction of crack propagation. Journal of Structural Engineering, 26, 1999, pp. 39-48

[4] Lewicki, D. G.: Crack Propagation Studies to Determine Benign of Catastrophic Failure Modes for Aerospace Thin-Rim Gears. NASA Technical Memorandum 107170, Cleveland, NASA, 1996

[5] Lewicki, D. G., Sane, A. D., Drago, R. J., Wawrzynek, P. A.: Three-Dimensional Gear Crack Propagation Studies. NASA Technical Memorandum 208827, Cleveland, NASA, 1998

[6] Franc2d, A Two Dimensional Crack Propagation Simulator, User's Guide, Version 2.7. New York, Cornell University, 1998

[7] Franc3d, A Three Dimensional Fracture Analysis Code, Concepts and User's Guide, Version 1.14. New York, Cornell University, 1998

[8] MARC Volume A Manual: Theory and User Information, Version K7. Los Angeles, MARC Analysis Research Corporation, 1997

[9] Dodds, R. H. Jr., Vargas, P. M.: Numerical evaluation of domain and contour integrals for nonlinear fracture mechanics: formulation and implementation aspects. Illinois, University of Illinois at Urbana-Champaign, 1988

[10] Shih, C. F., de Lorenzi, H. G., German, M. D.: Crack Extension Modelling with Singular Quadratic Isoparametric Elements. Int. J. Fracture, 12, 1977, pp. 647-651

[11] Mi, Y.: Three-Dimensional Analysis of Crack Growth. Southampton, Computational Mechanics Publications, 1996 
[12] Barsoum, R. S.: On the use of isoparametric finite elements in linear fracture mechanics. International Journal for Numerical Methods in Engineering, 10, 1976, pp. 25-37

[13] Sähn, S., Göldner, H.: Bruch- und Beurteilungskriterien in der Festigkeitslehre. Leipzig, Fachbuchverlag, 1989

[14] Bittnar, Z., Šejnoha, J.: Numerické metody mechaniky 2. Praha, Vydavatelství ČVUT, 1992

[15] Dhondt, G.: Mixed-Mode calculations with ABAQUS. In: "DVM Bericht 232: Festigkeits- und Bruchverhalten von Fügeverbindungen”, Berlin, DVM 2000, pp. 333-343

[16] Bažant, Z. P., Planas, J.: Fracture and size effect in concrete and other quasibrittle materials. Boca Raton, CRC Press, 1998

[17] Shivakumar, K. N., Raju, I. S.: Treatment of singularities in cracked bodies. International Journal of Fracture, 45, 1990, pp. 159-178

Dipl.-Ing. Michal Vorel

phone: +4903715314566

fax: +4903715314560

e-mail: michal.vorel@mbv.tu-chemnitz.de

Prof. Dr. Ing. Erhard Leidich

Technical University Chemnitz

Faculty of Mechanical Engineering

and Process Technology

Reichenhainerstraße 70

Chemnitz, D-09126, Germany 